\title{
Ist ein Verbot der NPD sinnvoll? Die Pioniere der deutschen Parteientheorie im Vormärz wären skeptisch
}

\author{
Philipp Erbentraut
}

\section{Verbieten oder tolerieren - eine alte Debatte neu aufgerollt}

Die NPD verbieten oder mit Rechtsextremen leben? Diese jahrelange Debatte hat seit Bekanntwerden der ideologisch motivierten Mordserie durch den so genannten Nationalsozialistischen Untergrund (NSU) und der Einsetzung eines Parlamentarischen Untersuchungsausschusses zur Aufklärung der schweren Straftaten neue politische Sprengkraft erhalten. Jenseits der konkreten rechtlichen Bedingungen und Möglichkeiten eines Verbotsverfahrens steht dabei die Frage nach dem generellen Sinn oder Unsinn von Parteiverboten innerhalb des demokratischen Verfassungsstaates im Zentrum der Kontroverse.

Interessanterweise wird dabei in der gegenwärtigen Diskussion - zumeist unwissentlich - ein fast 200 Jahre alter Diskurs reproduziert. Parteiverbote werden in Deutschland nämlich nicht erst seit den Gründerjahren der Bundesrepublik und den beiden erfolgreich abgeschlossenen Verfahren gegen die SRP 1952 und die KPD 1956 erörtert. Schon die vermeintlich so wehrlose Demokratie der Weimarer Republik kannte dieses Instrument und machte reichlich Gebrauch davon. Auf Grundlage des Gesetzes zum Schutze der Republik, des Reichsvereinsgesetzes sowie verschiedener Ausnahmeverordnungen wurden in dieser Zeit nicht weniger als 28 Verbote gegen politische Parteien ausgesprochen. Nicht zuletzt wurde im Jahr 1923 die NSDAP im gesamten Reichsgebiet aufgelöst. ${ }^{1}$

Indes: Parteiverbote stellen, noch viel früher, bereits in der Vor- und Frühgeschichte des deutschen Parlamentarismus vor 1848 einen wichtigen Gegenstand der staatstheoretischen Auseinandersetzung dar. Diese Entdeckung bedeutet nicht allein ein wissensarchäologisches Kuriosum. Der Blick in das ideengeschichtliche Archiv der deutschen Staatsphilosophie des Vormärz könnte vielmehr zur Aufstockung des argumentativen Arsenals in der heutigen Parteiverbotsdebatte einen wertvollen Beitrag leisten.

Umso erstaunlicher ist dieser Umstand, da es in Deutschland vor 1848 überhaupt noch keine Parteien, sondern allenfalls „apokryphe Formen des politischen Parteiwesens“2 gab. Zumindest existierten Parteien mangels konstitutioneller Anknüpfungspunkte nicht im heutigen Verständnis als politische Organisationen, die sich an Wahlen beteiligen. Auf gesamtdeutscher Ebene gab es bis zur Nationalversammlung in der Frankfurter Paulskirche kein Parlament. Wo sollten die Parteien hier ein praktisches Betätigungsfeld finden? So verstanden die Zeitgenossen vor 1848 unter Parteien in aller Regel keine realen politischen Gruppen, sondern ideologische Gesinnungsgemeinschaften, Schulen oder gesellschaftliche Großströmungen. ${ }^{3}$ Bestrebungen, die auf eine Verfestigung der Organisationsstrukturen zielten, waren

1 Vgl. Katrin Stein, Parteiverbote in der deutschen Verfassungsgeschichte vom Vormärz bis zum Ende der Weimarer Republik, in: ZParl, 32. Jg. (2001), H. 3, S. 536 - 550, S. 546 f.

2 Ernst RudolfHuber, Deutsche Verfassungsgeschichte seit 1789, Bd. 2: Der Kampf um Einheit und Freiheit 1830 bis 1850 , Stuttgart u.a. 1988, S. 319.

3 Vgl. Klaus von Beyme, Partei, Faktion, in: Otto Brunner / Werner Conze / Reinhart Koselleck (Hrsg.), Geschichtliche Grundbegriffe. Historisches Lexikon zur politisch-sozialen Sprache in Deutschland, 
nach dem Hambacher Fest 1832 im gesamten Deutschen Bund verboten. ${ }^{4}$ Zuvor hatten bereits die Karlsbader Beschlüsse von 1819 mit ihren weitreichenden Grundrechtsbeschränkungen einem freien Parteiwesen den Boden entzogen. Der monarchisch-bürokratische Staat fürchtete um seine Einheit und Geschlossenheit und verhielt sich feindselig gegenüber allen Parteien. Selbst die Verherrlichung ausländischer Parteien stand unter Strafe. ${ }^{5}$

Trotz oder vielleicht gerade wegen dieser unwirtlichen äußeren Umstände entwickelte sich vor allem in den 30 er und 40er Jahren des 19. Jahrhunderts eine äußerst fruchtbare und phantasievolle Debatte um den zukünftigen Standort politischer Parteien in der (damals noch zu schaffenden) Verfassung. Pointiert könnte man von Parteientheorien avant la lettre sprechen. In diesem Zusammenhang tauchte erstmals auch die Frage auf, wie ein insgesamt parteienfreundlich gewordener Staat in der Zukunft mit Verfassungsfeinden verfahren solle. Quer durch alle politischen Lager überwog dabei in Sachen Parteiverbot die Skepsis.

Die Vielfalt der vorgebrachten Argumente lässt sich in drei Hauptthesen bündeln, die auf verblüffende Weise alle heute relevanten Bedenken vorweg zu nehmen scheinen ${ }^{6}$ : die Vergeblichkeitsthese, die Gefährlichkeitsthese und die Sinnverkehrungsthese. Kurzgefasst lauten die jeweiligen Einwände: Parteiverbote nützen nichts (Vergeblichkeit), sind schädlich (Gefährlichkeit) und widersprechen dem Geist der Demokratie (Sinnverkehrung). Die Thesen, die ursprünglich vom amerikanischen Soziologen Albert O. Hirschman in einem anderen Kontext entwickelt wurden ${ }^{7}$, sind idealtypisch zugespitzt. Natürlich finden sich in den Stellungnahmen der meisten Autoren - damals wie heute - Kombinationen aller drei Erklärungsmuster, hauptsächlich wohl mit dem Ziel einer additiven Verstärkung. Streng genommen sind aber nicht alle Annahmen beliebig miteinander kompatibel. So könnte man in der Sinnverkehrungsthese eine Übertreibung oder einen Spezialfall der Gefährlichkeitsthese sehen, die gesondert auf die potenziell schädlichen Auswirkungen eines Parteiverbots für die Demokratie selbst abhebt. Man kann aber logischerweise nicht gleichzeitig behaupten, dass Parteiverbote schädlich und wirkungslos auf einmal sind. Wie argumentieren die Pioniere der deutschen Parteientheorie nun im Einzelnen?

\section{Die Vergeblichkeitsthese: "Ist Euch eine Partei lästig, zeigt ihre Unwahrheit auf“}

Zu den profiliertesten Anhängern der Vergeblichkeitsthese zählte im Vormärz der Junghegelianer Arnold Ruge, der für die unbeschränkte Betätigungsfreiheit aller Parteien eintrat:

Bd. 4, Stuttgart 1978, S. 677 - 733; Theodor Schieder, Die Theorie der Partei im älteren deutschen Liberalismus, in: ders., Staat und Gesellschaft im Wandel unserer Zeit, München 1974, S. 110 - 132.

4 Vgl. den zweiten Bundesbeschluss ,über Maßregeln zur Aufrechterhaltung der gesetzlichen Ruhe und Ordnung im Deutschen Bunde" vom 5. Juli 1832, in: Ernst Rudolf Huber (Hrsg.), Dokumente zur deutschen Verfassungsgeschichte, Bd. 1: Deutsche Verfassungsdokumente 1830-1850, Stuttgart u.a. 1978, S. 134.

5 Vgl. Preußische Zensur-Verordnung vom 18. Oktober 1819, in: ebenda, S. 106.

6 Für einen Überblick über die aktuelle Verbotsdebatte vgl. Uwe Backes, NPD-Verbot: Pro und Contra, in: APuZ, 62. Jg. (2012), H. 18/19, S. 9 - 15; Eckart Klein, Ein neues NPD-Verbotsverfahren? Rechtsprobleme beim Verbot politischer Parteien, Baden-Baden 2012. Vgl. ferner zur Diskussion im Vorfeld des gescheiterten NPD-Verbotsverfahrens im Jahr 2003: Claus Leggewie I Horst Meier (Hrsg.), Verbot der NPD oder Mit Rechtsradikalen leben? Die Positionen, Frankfurt am Main 2002.

7 Vgl. Albert O. Hirschman, Denken gegen die Zukunft. Die Rhetorik der Reaktion, München 1992. 
„Partei! Man erschrickt, wenn dies schreckliche Wort in einem vernünftigen Zusammenhange auftritt; denn über nichts ist wohl so oft schon größres Lamento erhoben worden als eben über das Parteiwesen - dieses ,Unwesen'. "8 Parteien die volle politische Freiheit zu gewähren, sei allerdings ganz das, „was das Freigeben der geistigen Gegensätze in der Wissenschaft, die Freiheit der Forschung und der Geltendmachung des Gedankens in der Theorie ist“. In der frei gesetzten Parteibewegung könne die „,befruchtende Macht der Negativität“ geschichtlich wirksam werden, schrieb der radikale Demokrat 1842. Ängstliche Gemüter versuchte er zu beruhigen. Das Lob der Partei sei in Wahrheit „nicht eine Anleitung zur Revolution, sondern eine Angabe, wie sie gründlich zu vermeiden sei“"?

Anstatt auf staatliche Restriktionen zu setzen, empfiehlt Ruge gegenüber extremen Positionen, die theoretische und gesellschaftliche Auseinandersetzung zu forcieren. Eine Partei hat für ihn ohnehin nur dann Aussicht auf Erfolg, wenn sie gewissermaßen die „Macht der Zeit“ repräsentiert. Da aber niemand den Zeitgeist manipulieren kann, sind Parteiverbote sinnlos. Darum könne man alle Einseitigkeiten ruhig gewähren lassen: „Ist ihre Zeit vorüber, oder haben sie gar niemals eine Zeit gehabt, so werden sie am sichersten sich selbst widerlegen. Ist das nicht der Fall, so sind sie eben an der Zeit." Schläge gegen die organisatorische Basis verpuffen. Zum Sieg führt allein der zwanglose Zwang des besseren Arguments. „Bloße Gewalt, und wäre sie überdem noch so schlau [...], gelangt nicht zum Ziele." Wie auf die NPD gemünzt klingt sein Fazit: „Ist Euch daher eine Partei lästig, so zeigt ihre Unwahrheit auf, und ihre Lebensader ist auf immer zerschnitten. "10

Auch Ruges Gesinnungsgenosse, der Radikaldemokrat und Publizist Wilhelm Schulz, gab sich als Anhänger einer ausgesprochenen Laisser-faire-Haltung zu erkennen. Seine einschlägigen Beiträge aus den Jahren vor der Revolution sind darüber hinaus ein Paradebeispiel dafür, wie der Parteibegriff - dieses „schreckliche Wort“ - in Teilen der deutschen Staatsphilosophie und Publizistik allmählich neutraler aufgefasst oder sogar positiv konnotiert wurde, während die „Faktion“ - lange Zeit ein Synonym für Partei - ihren schlechten Klang beibehielt. Ihr gegenüber versuchte vor allem die politische Linke die Parteien nun als geeignete Vehikel zur Verwirklichung der Volkssouveränität in Stellung zu bringen, während der semantische Giftmüll, der sich über die Jahrhunderte am Parteibegriff abgelagert hatte, auf die Faktionen abgewälzt wurde. Aber selbst derart kontaminierte Vereinigungen wollte Schulz noch in Ruhe gewähren lassen.

„Eine Faction“, so definierte er, sei zwar eine Gruppe, „die ihre gemeinschaftlichen politischen Interessen im Staate mit hartnäckiger und leidenschaftlicher Thätigkeit verfolgt oder behauptet.“ Gemeinhin werde mit diesem Begriff „etwas Verwerfliches bezeichnet“. Der „Factionsgeist“ sei nämlich ausgesprochen „aufrührerisch und empörerisch“ und schrecke bei der Wahl seiner Mittel auch vor Gewalt nicht zurück. ${ }^{11}$ Allerdings könnten Parteien unter gewissen Umständen zu Factionen degenerieren. Unterdrückung sei jedoch keine Lösung: „Vergebliche Anstrengungen! Aber man erstickt nicht die Leidenschaften, wenn

8 Arnold Ruge, Kritik und Partei. Der Vorwurf gegen die neueste Geistesentwicklung, in: Deutsche Jahrbücher für Wissenschaft und Kunst, 5. Jg. (1842), S. 1175 - 1182, S. 1179.

9 Ebenda, S. 1182.

10 Ebenda, S. 1180.

11 Wilhelm Schulz, Faction, in: Carl von Rotteck / Carl Theodor Welcker (Hrsg.), Das Staats-Lexikon. Encyklopädie der sämmtlichen Staatswissenschaften für alle Stände, in Verbindung mit vielen der angesehensten Publicisten Deutschlands, Bd. 4, Altona 1846, S. 576 - 581, S. 576. 
man sie zur scheinbaren Ruhe zwingt und zum Schweigen verdammt, bis sie sich zum äußersten Grade gesteigert haben, bis aus der stillen Schwüle der verheerende Sturm und der zerschmetternde Strahl mit doppelt überraschender Gewalt hervorbrechen. "12

Ähnlich argumentierte 1843 der liberale Philosoph Karl Rosenkranz gegen jeden Versuch der Regierung, eine unbequem werdende Opposition mit Gewalt oder Verboten zu unterdrücken. Denn die Folge davon sei unfehlbar „das Entstehen geheimer Gesellschaften, welche den Parteizweck im Stillen weiter verfolgen“. Eine Partei, „welche aus substantiellem Boden entsprossen ist", könne auf diese Weise überhaupt nicht vernichtet, sondern ihre Entwicklung höchstens verzögert werden, allerdings um den Preis, dass sie „fanatisch“ wird. ${ }^{13}$

Über die Existenz solch fanatisierter Geheimbünde im vormärzlichen Deutschland wurde erbittert gestritten, vor ihrer Ausbreitung im Allgemeinen gewarnt. Ihre Entstehung, meinte Wilhelm Schulz, sei letztlich jedoch die traurige Konsequenz verweigerter Grundrechte. Die Regierung müsse sich an die eigene Nase fassen. Überall dort nämlich, wo die Verfassungen nicht so weit auf das Prinzip der Öffentlichkeit und politischen Freiheit gegründet seien, um allen Parteien und Meinungen eine verhältnismäßige Vertretung und die Mittel zu gewähren, sich in gesetzmäßiger Weise auszusprechen und geltend zu machen, „werden dann auch wohl geheime Verabredungen getroffen, Plane entworfen und es wird vielleicht zur Ausführung geschritten werden"14.

Auf diese Weise seien Geheimgesellschaften zwar allein schon durch ihre Existenz eine Provokation für die jeweiligen Machthaber. Ganz im Sinne der Vergeblichkeitsthese warnte Schulz dennoch vor restriktiven Schritten und plädierte stattdessen für eine liberale Präventionsstrategie: „Bloße Strafgesetze haben jedoch stets den beabsichtigten Erfolg verfehlt und nur da haben geheime politische Vereine und Umtriebe gar nicht oder selten aufkommen können, wo Freiheit der Presse und Associationsrecht vollständig anerkannt waren, darum geheime Verbindungen als überflüssig erscheinen mußten. “15

Indes gelte es die Entstehung von Geheimgesellschaften „als ein Symptom des Volkslebens sehr zu beachten“. Sie seien zumeist „Anzeichen einer wachsenden Opposition“, mit deren polizeilicher Unterdrückung gar nichts erreicht sei, „so lange nicht die Quelle der Unzufriedenheit, woraus sie entsprungen sind, versiecht ist. Die politischen Parteien haben noch lange nicht aufgehört, wenn man auch alle geheimen politischen Gesellschaften gesprengt hat; und gegen den Sturm, welchen diese, wenn nicht erzeugen, doch häufig ankündigen, giebt es stets nur ein sicheres Mittel: die Popularisierung der Verfassungen und der Gesetzgebung unter dem freien Einflusse der öffentlichen Meinung"16.

Bereits 1815 hatte der bekannte Altertumsforscher Barthold Georg Niebuhr zwischen legitimen politischen Parteien unterschieden, die im Lichte der Öffentlichkeit naturwüchsig in jedem gesunden Staat entstehen müssten und verschwörerischen Verbindungen, die für ihre Zusammenrottungen den Schutz der Dunkelheit suchten. Letztere stellten eine Verlet-

12 Ebenda, S. 580.

13 Karl Rosenkranz, Über den Begriff der politischen Partei. Rede zum 18. Januar 1843 am Krönungsfeste Preußens in der Königl. Deutschen Gesellschaft zu Königsberg, in: Hermann Lübbe (Hrsg.), Die Hegelsche Rechte, Stuttgart-Bad Cannstatt 1962, S. 65-85, S. 83 f.

14 Wilhelm Schulz, Geheime Gesellschaften, in: Carl von Rotteck / Carl Theodor Welcker (Hrsg.), a.a.O. (Fn. 11), Bd. 5, Altona 1847, S. 427 - 454, S. 447.

15 Ebenda, S. 450.

16 Ebenda, S. 451. 
zung der souveränen Macht dar und seien deshalb als Hochverrat zu bestrafen. Allen anderen politischen Unternehmungen gegenüber riet Niebuhr dagegen ebenfalls zur Gelassenheit. Wer nur diejenigen Parteien dulden wolle, „die ohne Fehl auf das Gute, Rechte und Wahre allein gerichtet sind, und dabei keinen einzigen falschen Bruder zählen, der weiß wenig wie es mit der Parthei beschaffen ist, zu der er selbst gehört" ${ }^{\text {"17. }}$.

\section{Die Gefährlichkeitsthese: Radikale als rettende Rauchmelder der Demokratie?}

Neben solch altersmilden oder fatalistischen Stimmen findet man in der verbotsskeptischen Literatur verschiedene Klugheitsargumente. So gehen die Vertreter der Gefährlichkeitsthese zwar von der generellen Berechtigung des Staates aus, Parteien notfalls zu verbieten. Auch wird nicht in Zweifel gezogen, dass Parteiverbote überhaupt eine Wirkung entfalten - wenn auch bisweilen die falsche. Bei diesen Autoren überwiegt jedoch die Sorge vor den unvorhersehbaren und nicht intendierten Konsequenzen eines Verbots und einer daraus resultierenden möglichen Verschlimmerung der Lage.

Auch unter diesem Blickwinkel spielte das Thema Parteiverbote ab Mitte der 1830er Jahre in der Bibel des deutschen Frühliberalismus, dem Rotteck-Welckerschen Staatslexikon, eine immer prominentere Rolle. So sprach sich Carl Theodor Welcker, einer der beiden Herausgeber, zwar ebenfalls für die generelle Freiheit zur Bildung politischer Vereine aus, wie man die Parteien damals meist nannte. Gleichwohl musste er gegenüber den Kritikern der organisierten Sonderinteressen eine "gewisse allgemeine Gefährlichkeit der Associationsfreiheit" ${ }^{18}$ durchaus einräumen. Manche Vereine könnten in der Tat eine sehr schlimme Richtung annehmen und der Staatsgesellschaft insgesamt Schaden zufügen. Solche vereinzelten und vorübergehenden Störungen könnten in der Gesamtbilanz aber nicht den bleibenden Wert des Gutes Vereinigungsfreiheit aufwiegen. Sollte es aus den Parteien heraus dennoch einmal zu Rechtsverletzungen kommen, regte der Autor an, die konkreten Handlungen einzelner Mitglieder zu bestrafen und nicht pauschal die gesamte Organisation zu verbieten. ${ }^{19}$ Zur Abwendung von unmittelbarer Gefahr seien vorübergehende Einschränkungen der Vereinigungsfreiheit im Notfall dennoch zulässig. Parteiverbote sollten aber nicht einseitig von der Regierung verhängt werden, sondern bedürften der parlamentarischen Kontrolle. ${ }^{20}$

Kein Freund staatlicher Zwangsmaßnahmen war auch der sozialkonservative Staatstheoretiker und Publizist Victor Aimé Huber, obwohl er den Aufstieg des vormärzlichen Liberalismus vor der 1848er Revolution mit großer Sorge verfolgte und sich gegenüber der gut geölten Propagandamaschine der politischen Linken bereits in der Defensive wähnte. Dennoch hielt Huber Verbote für unklug. Parteifreunde, die davon träumten, schimpfte er aus. Sie gäben sich einer „süßen Täuschung "21 hin. Damit sei gar nichts auszurichten. Verbote

17 Barthold Georg Niebuhr, Ueber geheime Verbindungen im preußischen Staat und deren Denunciation, Berlin 1815, S. 9.

18 Carl Theodor Welcker, Association, in: Carl von Rotteck / Carl Theordor Welcker (Hrsg.), a.a.O. (Fn. 11), Bd. 2, Altona 1835, S. 21 - 53, S. 43.

19 Vgl. ebenda, S. 33.

20 Vgl. ebenda, S. 52.

21 Victor Aimé Huber, Die Opposition. Ein Nachtrag zu der conservativen Parthei, Halle 1842, S. 46. 
erlaubten dem Gegner nur, sich als Opfer autoritärer Unterdrückung zu stilisieren und somit weitere Anhänger zu mobilisieren. Staatliche Restriktionen gewährten den Feinden der Verfassung den unermesslichen Vorteil, „sich vor der öffentlichen Meinung als Helden und Märtyrer der Freiheit spreitzen zu können und ihren Gegnern das Odium zuzuweisen, als scheuten sie die freie Diskussion aus irgend einem Grunde" 22 . Sinnvoller sei es stattdessen, die direkte politische Konfrontation zu suchen, um die breite Öffentlichkeit für die Positionen der Regierung zu gewinnen.

Dergestalt baute übrigens auch Carl Theodor Welcker auf einen Aufstand der Anständigen. Dazu sei allerdings ein liberaler, offener, ja listig-souveräner Umgang mit den Feinden der Verfassung geboten. Über kurz oder lang, so die Hoffnung, würden sich die geistigen Brandstifter dann selbst demaskieren: „Das Verkehrte aber und das der öffentlichen Ruhe wirklich Gefährliche kann die Regierung nunmehr nicht blos selbst offen angreifen und verhindern, sondern indem es offenbar wird, gewinnt sie auch alle ruheliebenden Bürger zu den kräftigsten Kämpfern dagegen und zu williger Unterstützung etwa nöthiger Gegenmaßregeln."23 Staatsgewalt und Zivilgesellschaft galten ihm als natürliche Bündnispartner im Kampf gegen den Extremismus.

Interessanterweise kommt Welcker in diesem Zusammenhang bereits auf das Problem der V-Leute zu sprechen. In einigen Ländern würden staatlich alimentierte agent provocateurs in unliebsame Vereinigungen eingeschleust, um dort gezielt Exzesse hervorzurufen und zu befördern. Auf diese Weise solle die öffentliche Meinung zu Gunsten der Regierung beeinflusst werden. Der Verfasser sieht solche Manöver, obwohl äußerst wirksam, durchaus kritisch. Sie dienten nicht der Aufklärung, sondern in erster Linie der „Befestigung und Beförderung der absoluten Regierungsgewalt “24. In diesem Sinne würde einer der wichtigsten deutschen Staatstheoretiker des 19. Jahrhunderts heute wahrscheinlich dafür plädieren, die V-Männer in den Ruhestand zu versetzen. Dieser Ratschlag scheint besonders hellsichtig angesichts der im Zuge der NSU-Aufarbeitung bereits zutage getretenen Durchsetzung der rechtsextremen Szene mit V-Leuten des Verfassungsschutzes, die - ungeachtet allen (un) beabsichtigten Schredderns von Beweisakten - noch dramatischer gewesen zu sein scheint, als vor dem letzten NPD-Verbotsantrag, der 2003 genau aus diesem Grund scheiterte. Dabei warnte bereits im Jahr 1816 ein besorgter Anonymus vor den unheilvollen Konsequenzen einer solchen Verquickung von "Staats-Polizey“ und extremistischen Vereinigungen: Würde die Regierung einer latenten Gefahr dadurch vorbeugen wollen, so der unbekannte Autor, „daß sie mehrere von ihren Staatsdienern an einer solchen geheimen Gesellschaft als Mitglieder Antheil nehmen ließe, um auf diesem Wege die Zwecke und die Operationen der Gesellschaft zu erfahren oder gar zu leiten, so wäre dies theils unter ihrer Würde, theils würde sie dadurch auch den nothwendigen Charakter der Oeffentlichkeit, und somit auch das öffentliche Vertrauen verlieren, welches doch die Basis der Selbstständigkeit des Staates ist. " 25 Treffender als in dieser alten Prophezeiung lässt sich das aktuelle Unbehagen an den

22 Victor Aimé Huber, Ueber die Elemente, die Möglichkeit oder Nothwendigkeit einer konservativen Parthei in Deutschland, Marburg 1841, S. 67.

23 Carl Theodor Welcker, a.a.O. (Fn. 18), S. 49.

24 Carl Theodor Welcker, a.a.O. (Fn. 18), S. 50.

25 Anonym, Ueber die Gefahren heimlicher Verbindungen, und über die Unzuläßigkeit geheimer Gesellschaften im Staate, in: Allemannia. Für Recht und Wahrheit, 5. Jg. (1816), S. 185 - 224, S. $199 \mathrm{f}$. 
zwielichtigen Kontakten des deutschen Verfassungsschutzes wohl nicht auf den Punkt bringen.

Schließlich wurde ein weiterer kritischer Gesichtspunkt in der Parteiverbotsdebatte von denjenigen Theoretikern hervorgehoben, die im Aufkommen extremistischer Parteien eine Art Rauchmelderfunktion oder ein politisches Frühwarnsystem vor drohenden revolutionären Tsunamis erblickten. So sah Ludwig Bubl die Parteien seiner Zeit in einem ständigen, Staat und Gesellschaft befriedenden Abschleifungs- und Vermittlungsprozess begriffen: „Wo die Parteien nicht vertreten sind, wo sie sich nicht gegeneinander abreiben können, da ist immer eine gewaltsame Explosion des unterdrückten Gegensatzes zu fürchten. “26 Die „Ventile an dem Dampfkessel, in dem der Volksgeist siedet "27 zuzudrehen, könnte demnach katastrophale Folgen haben.

Andere Autoren bemühten organologische Metaphern aus dem Bereich der Körperhygiene. Extremistische Parteien seien nicht das eigentliche Übel, sondern lediglich Symptome einer tiefer liegenden, ansonsten unbemerkten Krankheit des Staatskörpers. Die Geschwüre zu beseitigen, sei noch keine Heilung. ${ }^{28}$ Nicht zuletzt wurde einseitige staatliche Einmischung in den Parteienwettstreit immer wieder mit Verweis auf die tradierten Turnierregeln mittelalterlicher Ritterspiele zurückgewiesen. Die Regierung habe dafür Sorge zu tragen, dass jeder Partei „freies Feld und keine Gunst“29, „gleiche Waffen und gleiche Sonne“30 zuteil werde. Keinesfalls dürfe sie selbst Partei nehmen. Andernfalls riskiere der Staat das Vertrauen und die Liebe zumindest eines Teils seiner Bürger.

\section{Die Sinnverkehrungsthese: Verbote zerstören, was sie zu schützen beabsichtigen}

Vor allem der letztgenannte Punkt trieb auch die Verkünder der Sinnverkehrungsthese um. Der Kerngedanke lautet hier, dass Parteiverbote einen systemwidrigen Fremdkörper im demokratischen Prozess darstellen, die mithin dem Geist der Demokratie zuwiderlaufen. Eine freiheitliche Ordnung kann demnach Parteien als wichtigste Akteure des politischen Meinungs- und Interessenpluralismus nicht verbieten, ohne Gefahr zu laufen, selbst autoritär zu werden und somit letzten Endes genau jenes Gut zu zerstören, das sie zu schützen beabsichtigt. Um ihrer selbst willen müsse die Demokratie deshalb mit Verfassungsfeinden leben. Fast beschwörend meinte zum Beispiel Welcker: Tüchtige Staatsmänner und Regierungen „werden nicht wegen des möglichen einzelnen Mißbrauchs der Freiheit die Freiheit selbst aufgeben wollen" 31 .

Der wichtigste Vertreter der Sinnverkehrungsthese im Vormärz war der Barrikadenkämpfer und spätere demokratische Paulskirchenabgeordnete Julius Fröbel, der mit Hilfe der Parteien den einmaligen historischen Gründungsakt der Verfassung in Form einer „legalen und

26 Ludwig Buhl, Der Beruf der Preußischen Presse, Berlin 1842, S. 20.

27 Arnold Ruge, Was wird daraus werden? Ein politischer Brief an die Deutschen, in: ders. (Hrsg.), Die Akademie. Philosophisches Taschenbuch, Bd. 1, Leipzig 1848, S. 191 - 226, S. 203 f.

28 Vgl. Victor Aimé Huber, a.a.O. (Fn. 22), S. 40.

29 Karl Rosenkranz, a.a.O. (Fn. 13), S. 72.

30 Anonym [Karl Reinhold Jachmann?], Preußen seit der Einsetzung Arndt's bis zur Absetzung Bauers, in: Georg Herwegh (Hrsg.), Einundzwanzig Bogen aus der Schweiz, Zürich / Winterthur 1843, S. $1-32$, S. 15.

31 Carl Theodor Welcker, a.a.O. (Fn. 18), S. 43. 
permanenten Revolution“32 auf Dauer stellen wollte. Fröbel setzte auf die vollkommene Freiheit der persönlichen Meinungsäußerung und der theoretischen Propaganda. „Selbst wenn der Zweck gegen die Existenz des Staates gerichtet wäre, sollte der theoretische Betrieb frei sein. Man kann der Meinung sein daß es besser wäre der Staat dessen Glied man ist bestünde gar nicht, [...] und diese Meinung muß erlaubt sein, und für sie muß man Theilhaber suchen dürfen. Eine Partei mit solchen Tendenzen wäre freilich ein innerer Feind, aber nur ein theoretischer, der also auch nur theoretisch zu bekämpfen wäre."33

Eine verfassungsfeindliche Haltung allein legitimiert für Fröbel also noch nicht den Einsatz staatlicher Zwangsmittel, selbst wenn die betreffende Partei ihre abweichenden Positionen in aktiv-kämpferischer, aggressiver Pose vorträgt. Erst eine Organisation, die zur Durchsetzung ihrer Ziele zum praktischen Mittel der Gewalt greift, tritt dadurch in das Verhältnis „eines Staatsfeindes und muß als solcher behandelt werden“34. Anders als das Bundesverfassungsgericht heute verlangt Fröbel anno 1847 also eine konkrete Gefahr für die demokratische Gesellschaft, mithin eine realistische Chance der Machtübernahme durch die feindliche Partei. Er liegt somit eher auf der Linie der gängigen Entscheidungspraxis des Europäischen Gerichtshofes für Menschenrechte in Straßburg, der ein mögliches deutsches NPD-Verbot nachträglich kassieren könnte.

Ein formales Parteiverbot scheint Fröbel aber selbst in einer solchen Ausnahmesituation nicht adäquat. Durch den Einsatz von Gewalt begebe sich die gegnerische Partei außerhalb der sittlichen Ordnung des Staates. Und somit gelte „kein Criminalrecht sondern Kriegsrecht “35. Fröbel fürchtet offenbar um die Integrität der Justiz. Diese könnte sich in einem politischen Schauprozess die Hände schmutzig machen und ihre ureigensten Prinzipien desavouieren. Vor welches Gericht will man den Staatsfeind stellen? „Wollte man ihn strafrechtlich behandeln, so müßte man ihn vor ein Forum ziehen welches er eben nicht anerkennt, und einem Gesetze unterwerfen welches er eben als untauglich erklärt hat." So würden der richterlichen Praxis die wichtigsten Erfordernisse ihrer Möglichkeit fehlen, meint der Autor. „Sie könnte nicht zwischen Parteien entscheiden, denn sie wäre selbst die eine Partei. Sie könnte nicht einen einzelnen Fall nach einer anerkannten Rechtsnorm entscheiden, denn die Rechtsnorm wäre eben der streitige Punkt." So würde der Rechtsstreit unweigerlich zum „sittlichen Principienstreit“. Für derlei Gegensätze gebe es aber keinen gemeinsamen Rechtsboden. ${ }^{36}$

Fröbel gibt außerdem zu bedenken: „In der Regel ist es nicht die aufständige sondern die herrschende Partei, welche gewaltthätig verfährt und sich in der Offensive befindet. "37 In jedem Fall sei der Staat gut beraten, „durch die theoretische Freiheit und die allgemeine Theilnahme an der Gesetzgebung den Parteien eine legale, in den Staatsorganismus eingereihte Existenz und Bewegung zu geben, und so die Revolution durch ihre Legalität und Permanenz unschädlich zu machen"38.

32 Julius Fröbel, System der socialen Politik, Bd. 2, S. 274.

33 Ebenda, S. 282.

34 Ebenda, S. 283.

35 Ebenda, S. 283.

36 Vgl. ebenda, S. 287.

37 Ebenda, S. 291.

38 Ebenda, S. 292. 


\section{Schlussbetrachtung: Was würden die Altvorderen zur NPD raten?}

Lässt sich aus diesen fast 200 Jahre alten Ideen noch Honig für die heutige Debatte saugen? Unter dem Strich war die deutsche Staatsphilosophie im Vormärz jedenfalls deutlich skeptisch, was den Sinn von Parteiverboten angeht. Einige Autoren hielten dieses Schwert schlicht für stumpf. Die meisten waren indes der Ansicht, dass Parteiverbote sehr wohl wirken, allerdings mehr Schaden anrichten als nützen. Nicht zuletzt schien manchem durch die Anwendung von staatlichen Zwangsmaßnahmen die Demokratie selbst in Gefahr. Dennoch wäre es voreilig, aus dem Spruch der Altvorderen den Schluss zu ziehen, man müsse dem Treiben der Rechtsextremen tatenlos zusehen. Forderungen nach mehr politischer Bildung oder argumentativer Widerlegung extremistischer Positionen zählten nämlich schon vor 1848 zum Katechismus des braven Republikaners. Auch nachdenklichere Töne wurden damals laut. Das Aufkommen feindlicher Parteien sei wohl kein Zufall, meinte ein angesehener zeitgenössischer Staatsrechtler, sondern deute in der Regel auf ein Versagen, ja „eine wunde Stelle“ in der eigenen Verfassung oder Regierung hin. Von dieser Seite könne man aber ,jenem Feinde, der im Herzen des Landes steht, meist am besten beykommen“39. Mit Reformen also, die Ursachen beheben statt Repressionen, die nur Symptome bekämpfen. Dieser aktiv gestalterische Spielraum der Demokratie kommt in der gegenwärtigen Debatte zu kurz. Er gerät in den Hintergrund angesichts einer aus Gründen tagespolitischer Opportunität unter dem Eindruck unmittelbarer gewalttätiger Aktionen immer wieder reflexartig artikulierten NPD-,,Verbotslust“ der Politik.

Die Pioniere der Parteientheorie im vormärzlichen Deutschland waren da selbstbewusster und auch ein Stück weit gelassener. Ihre Gedanken atmen noch mit jeder Faser den Geist einer Epoche, die von Recht und Gerechtigkeit der jeweils eigenen politischen Visionen vollkommen überzeugt war. Im unerschütterlichen Glauben an die prinzipielle Vernunft und Zivilcourage einer aufgeklärten Aktivbürgerschaft wäre damals kaum jemand auf die Idee gekommen, die Demokratie mit autoritären Mitteln schützen zu wollen. Man setzte stattdessen auf die politische Konfrontation mit offenem Visier. Für das Konzept der Streitbaren Demokratie sprechen in der Bundesrepublik historisch gewichtige Gründe. Im Lichte einer heute gefestigten Verfassung sollten wir die Attraktivität der freiheitlich-demokratischen Grundordnung aber vielleicht nicht unterschätzen. In diesem Sinne könnte das abschließende Credo der vormärzlichen Parteientheorie lauten: Stelle deine Feinde, anstatt sie zu unterdrücken!

39 Karl Salomo Zachariä, Vierzig Bücher vom Staate, Bd. 2, Stuttgart / Tübingen 1820, S. 70. 